\title{
Mastoid Obliteration using HA granules and pericranial flap : technique and results.
}

\author{
Wendy Smith ${ }^{1}$ and Swagatam Banerjee ${ }^{2}$ \\ ${ }^{1}$ Leeds Teaching Hospitals NHS Trust \\ ${ }^{2}$ University Hospitals Coventry and Warwickshire NHS Trust
}

July 7, 2020

\begin{abstract}
Introduction: The creation of the "open cavity" to clear cholesteatoma results in significant morbidity in over a third of patients. Various biological and non-biological materials have been used to reduce or obliterated these mastoid cavities. Materials and Methods: This study reports the technique and results of using hydroxyapatite granules with a pericranial flap to obliterate troublesome cavities. Results: 50 mastoid obliterations were performed in 48 patients. $94 \%$ no longer required water precautions 2 months post-operatively and may use conventional hearing aids. Discussion: This technique improves patients' quality of life and can enable discharge of patients previously reliant on ENT services.
\end{abstract}

\begin{abstract}
Introduction: The creation of the "open cavity" to clear cholesteatoma results in significant morbidity in over a third of patients. Various biological and non-biological materials have been used to reduce or obliterated these mastoid cavities.

Materials and Methods: This study reports the technique and results of using hydroxyapatite granules with a pericranial flap to obliterate troublesome cavities.

Results: 50 mastoid obliterations were performed in 48 patients. $94 \%$ no longer required water precautions 2 months post-operatively and may use conventional hearing aids.

Discussion: This technique improves patients' quality of life and can enable discharge of patients previously reliant on ENT services.
\end{abstract}

Keywords: mastoid obliteration hydroxyapatite granules cholesteatoma pericranial flap quality of life

Keypoints:

The use of HA granules has been described previously emphasising a thorough(revision) modified radical mastoidectomy before reconstruction is performed. This study however describes the following modifications of the technique:

- Optimise operative conditions with blood loss of 1-2ml with the anaesthestist to consider using remifentanyl, tranexaminc acid and hyperventilation of the patient so pCO2 is 4 to achieve this.

- No drain is required enabling this to be a daycase procedure

- Consider performing an anterior bony canalplasty to optimise middle ear surgery and prevent the ear canal becoming too narrow.

- In addition to the cartilage to form the "new "posterior ear canal wall, consider placing a separate piece of cartilage to block the attic. 
- Using this technique $94 \%$ patients no longer require water precautions and some may use a conventional hearing aid.

- This operation has a positive impact on quality of life as measure by Glasgow benefit inventory scores.

Introduction:

Since Gustave Bondy, an Austrian surgeon popularized the modified radical mastoidectomy technique in $1910,{ }^{1}$ the problems of the open cavity in some patients have been evident in ENT clinics. There is often a need for patients to take water precautions (avoiding swimming) and even following such advice some patient experience constant or intermittent otorrhoea. Many patients are reliant on their local ENT services for aural toilet (which can impact on work commitments). Some patients with hearing loss are unable to use conventional hearing aids either due to a poor fit and feedback occurring due to the associated meatoplasty, or as a result of the hearing aid increasing the humidity of the ear canal resulting in repeat infections. Other patients may experience a caloric effect as a result of the meatoplasty allowing cold air to stimulate an exposed semi-circular canal.

In 1911 Mosher described Plummer's technique of using a soft tissue flap to fill the excavated mastoid ${ }^{2}$. Many others including Popper, ${ }^{3}$ Meurman and Ojala, ${ }^{4}$ Guildford, ${ }^{5} \mathrm{Palva}^{6}$ and the Hong Kong flap ${ }^{7}$ have been described along with various fillers. Fat, cartilage, bone chips/pate have been used but they tend to resorb with time and although one could "overfill" to compensate, the results are unpredictable and some authors have raised concerns that using cortical bone could result in cholesteatoma becoming incorporated into the obliterated cavity. ${ }^{8}$ A number of non-biological materials have been tried including the use of titanium (covered with cartilage), ${ }^{9}$ bioglass,${ }^{10}$ hydroxyapatite (HA) ${ }^{11}$ and SerenoCem. ${ }^{12}$ The latter was introduced in 1999 but was taken off the market in 2017 due to concerns that it caused skull base erosion. Hydroxyapatite cement was associated with post-operative infections however the granules introduced by Takahashi in 1991 do not appear to have this issue. ${ }^{11}$ The technique and results of using HA granules, cartilage and pericranial flap based on the mid-temporal artery and vein is described.

Materials and methods: All 46 patients who had a secondary mastoid obliteration having failed active medical management of their problematic modified radical mastoidectomy cavities along with 4 patients having a modified radical mastoidectomy and primary obliteration were included in this study. Their data was contemporaneously collected on the Common Otology Database and further information collated with Glasgow Benefit Inventory questionnaires sent to the patients following their surgery.

Surgical technique:

The surgery was performed under general anaesthesia and the conditions optimised to minimise blood loss to 1-2 ml. This was achieved by the anaesthetist using remifentanil (most patients are young and so other techniques may result in a rebound tachycardia when hypotension is induced), tranexamic acid $1 \mathrm{~g}$ on induction noticeably reduced "ooze" after 20 minutes and by hyperventilating the patient so the partial pressure expired CO2 was only 4, further vasoconstriction was achieved.

Skin preparation was applied after tape was used to cover the hair avoiding the need for hair removal, an important factor for the patients.

A thorough modified radical mastoidectomy is performed ensuring cholesteatoma has been removed with particular attention to the sinodural angle, mastoid tip, around the semi-circular canals and the anterosuperior mesotympanum. Keeping any high facial ridge and using endoscopes to visualise the middle ear makes subsequent reconstruction easier.

The hydroxyapatite granules are soaked in an antibiotic solution (cefuroxine $750 \mathrm{mg}$ in $10 \mathrm{ml}$ water for injections) for at least 15 minutes.

Conchal cartilage is harvested. In addition to a large piece of cartilage used to form the new posterior canal wall, a separate smaller piece is used to block the ensuring good positioning to prevent the formation of recurrent cholesteatoma as shown in figures $1 \mathrm{a}, 1 \mathrm{~b}$ and 2 . 
The pericranial flap is then raised by lifting the temporalis muscle fibres off the pericranium and an anterior based flap is raised taking care not to cut the mid temporal artery and vein as in figures $3 \mathrm{a}$ and $3 \mathrm{~b}$. In order to gain "extra length" a channel as shown in figure 4 can be used.

Once the any pars tens perforation has been repaired with for example temporalis fascia the cartilage is placed and HA granules (having drained the antibiotic solution) are used to fill the mastoid cavity ensuring that they also fill the anterior attic.

The pericranial flap is then place between the cartilage and the fascia as shown in figures 5a and 5b.

The ear canal is then packed. The senior author prefers Betnovate $\mathrm{C}$ ointment ribbon gauze avoiding the use of BIPP to prevent the development of allergic reactions associated with BIPP.

The patient is usually allowed home after 2 hour observation period on the ward. The patient removes their head bandage the following day keeping the 2 packs in the ear canal for 10 days. This is replaced in the clinic with another pack that the patient removes a week later before commencing 5 days of antibiotic/steroid ear drops. The patient observes water precautions until 2 months after surgery when they are next reviewed with a hearing test. If healed no water precautions are required.

Patients are followed up at 6 months then yearly, having non-EPI DWI MRI scans at 1, 3 and 5 years before discharging patients from the clinic.

Results: 50 mastoid obliteration surgeries have been performed in 48 patients over the 9 year study period. There were 10 female and 38 male patients. Their age was 10-78 years with a median age of 34 years old. Operations were performed on 24 right and 26 left ears.

The main indication for surgery was discharging ears (31 intermittent had discharge, and 16 having constant discharge). Only 3 patients had surgery without having a history of ear discharge; 2 had surgery to stop their dizziness experienced in the wind (caloric effect) and the other patient had surgery for social reasons after having had a successful operation on the other ear.

Follow-up was 2 to 9 years with a median follow-up of 4.5 years and the typical post-operative appearance is shown in figures $6 \mathrm{a}, 6 \mathrm{~b} \& 6 \mathrm{c}$.

Table 1 shows the number of ears with perforated ear drums which may contribute to the ears discharging.

Table 2 shows the distribution of ears with and without the presence of cholesteatoma found intra-operatively.

Patients were followed up in the clinic to assess whether any cholesteatoma was evident. An anterior attic pocket where the cartilage blocking the attic had not reached the anterior attic wall led to recurrent cholesteatoma formation in one patient who underwent revision surgery to address this. One patient was found to have an area of restricted diffusion in the attic of his right ear detected at 3 years following his revision mastoidectomy and obliteration. (Figure 7).

To date 48 ears (all asymptomatic) have had non-EPI DWI MRI scans. 2 patients had failed to attend follow-up. 5 ears were reported to have cholesteatoma present on follow-up scans. Two ears were explored in a tertiary centre and cholesteatoma was only found in one of these (figure 7). 2 ears were explored by the senior author and in both only cholesterol granuloma was found. One patient has been monitored with serial non-EPI DWI MRI scans and the $4 \mathrm{~mm}$ area of restricted diffusion has remained the same size over 6 years.

There have been no facial nerve palsies or residual/new tympanic membrane perforations. One patient became dizzy 4 days post-surgery (cholesteatoma had eroded the labyrinth but no residual cholesteatoma was found when this ear was re-explored). Post-operatively some patients had some granulations, myringitis treated with silver nitrate cautery and mild otitis externa that were treated with topical antibiotic and steroid preparations. One patient had a polyp in the ear canal removed under a short general anaesthetic (patient factors prevented its removal in the clinic). One patient had excess skin to line the new ear canal 
and it took 4 months to settle completely. $94 \%$ of patients had their ears free from infection and no longer required any water precautions at 3 months post-operatively as demonstrated in figures $6 \mathrm{a}, 6 \mathrm{~b}, 6 \mathrm{c}$.

This primary aim of this surgery is not to improve hearing but to stop ear discharge and for social reasons. After surgery many patients were offered a conventional hearing aid but they decline this since their hearing loss was not an issue to them. In 30 of the 50 ears no attempt was made to reconstruct the hearing. In 14 ears cartilage alone was placed onto the stapes head to bridge the gap between the stapes head and the tympanic membrane. In 2 ears a titanium PORP and in 4 ears a titanium TORP covered with cartilage was used. The hearing at 2-3 months against the hearing pre-operatively is demonstrated in graph 1 . The line indicates what the hearing post-operatively would be if it remained the same as the pre-operative level. Interestingly the patient with the greatest improvement in their hearing had a TORP inserted.

Graph 2 shows the hearing results at 6 months. There appears to be a gradual drift towards the pre-operative hearing level perhaps as a result of a fibro-adhesive process in the middle ear.

Graph 3 shows the change in hearing in patients who had their hearing measured at 2-3 and 6 months. None had a significant loss of hearing post-operatively and in some patients the hearing improvement was translated to a clinically apparent gain.

Patients attending the clinic expressed the improvement to the quality of life that this surgical procedure had made and this is borne out by the values obtained in 35 patients who had completed the Glasgow Benefit Inventory. The results are shown in table 3 .

Discussion:

In 1996, Yung described the use of HA granules and an inferior based periosteal flap in 34 mastoid obliteration operations achieving trouble free ears in all except one patient due to incomplete epithelialization of the obliterated cavity. ${ }^{13}$ Munjal et al using the same technique with HA granules and pericranial flap and achieved trouble free ears in $84 \%$ of their 25 cases. They reported a $2.04 \mathrm{~dB}$ reduction in their AB gap. ${ }^{14}$

The majority (39/50) of the ears had no perforation indicating that the discharge was related to an unhealthy mastoid cavity. Cholesteatoma was present within the middle ear in a third of the ears with either frank cholesteatoma within the residual mastoid air cells or a significant build-up of keratin within the mastoid bowl with underlying unhealthy mucosa. 11 of 50 ears did have a perforation of the pars tensa and one could argue that this increase in the humidity within the outer ear could be addressed with a myringoplasty however performing the mastoid obliteration offers these patients a dry ear which does not require water precautions and may enable them to pursue water sports.

Yung described the use of high resolution CT scanning in detecting cholesteatoma in temporal bones obliterated with muscle and with HA granules. ${ }^{15}$ The contrast of the dark shadows produced by epithelial cysts contrasted to the white background of HA granules but not with the appearance of the muscle. One false positive occurred in 31 bones due to a cholesterol granuloma. Non -EPI DWI MRI scanning has replaced follow-up CT scanning as a means to detect residual cholesteatoma since CT scans only detected the presence of soft tissue/fluid to be present ${ }^{16}$. The SI values of cholesteatoma and HA granules were measured for the purpose of this study and found to be 1150-1525 Si and 500-600 SI respectively. Cholesteatoma has a value of 2-3 times greater than HA granules and can therefore be easily differentiated. To date 48 ears (all asymptomatic) have been scanned. 2 patients had failed to attend follow-up. The relatively high false positive reports of cholesteatoma being present have led to unnecessary further surgery in asymptomatic ears. Cholesterol granuloma was found and it is now the senior author's practice to rescan if a $4 \mathrm{~mm}$ or less area or restricted diffusion is found as well as reviewing the ADC, T1 and T2 MRI sequences which can differentiate cholesteatoma from other pathology.

The Glasgow Benefit Inventory scores for the patients in this study are comparable to those observed by Kurien who used autologous bone graft in both primary and secondary obliterations. ${ }^{17}$ In the secondary obliterations he found the Total Score to be 31, General Health 34, Social Support 39 and Physical Health of 25. Interestingly, he found that the patients who had mastoid obliteration as a secondary procedure had 
higher scores to those who had a primary obliteration. This may result from the more prolonged period of symptoms including foul smelling discharge from the ears prior to their surgery. Hurley and Iyer in 2020 also reported improved quality of life scores using GBI and COMBI questionnaire with the biggest improvement in resolution of smelly and discharging ears. ${ }^{18}$

References:

1. Richards GL. The so-called conservative mastoid operation, with a description of the technic of Heath, Bondy and Siebenmann. Annals of Otology Rhinology and Laryngology . 1911;20:578-94

2. Mosher HP. A method of filling the excavated mastoid with a flap from the back of the auricle. Laryngoscope 1911;21(12):1158-1163.

3. Popper O. Periosteal flap grafts in mastoid operations. South African Medical Journal. 1935;9:77-81

4. Meurman Y, Ojala L. Primary reduction of a large operation cavity in radical mastoidectomy with a muscle-periosteal flap. Acta Otolaryngology . 1949;37(3):245-52

5. Guildford F. Indications for obliteration and closed mastoid operations. Archives of Otolaryngology 1969;89(1):191-5

6. Palva T, Makinen J. The meatally based musculoperiosteal flap in cavity obliteration. Archives of Otolaryngology 1979;105(7):377-80

7. Hasselt CA ,Liu KC, Tong MC. The Hong Kong vascularised temporalis fascia flaps for optimal, mastoid cavity reconstruction. Rev Laryngology Otology and Rhinology (Bord) 1995;116(1):57-60

8. Roberson JB, Mason TP, Stidham KR. Mastoid obliteration: Autogenous cranial bone pate reconstruction. Otology and Neurotology2003;24:132-40

9. Schwager K, Zirkler J. Reconstruction of the mastoid using a titanium cage. Otology and Neurotology 2014;35(8):1463-5

10. Silvola JT. Mastoidectomy cavity obliteration with bioactive glass: A pilot study. Otolaryngology Head and Neck Surgery2012;147(1):119-26

11. Takahashi S. Tympanoplasty with mastoid obliteration using hydroxyapatite granules for aural cholesteatoma - a clinical and experimental study. Nihon Jibiinokoka Gakkai Kaiho1991;94(6):83342 [Abstract only. Full article in Japanese]

12. Harrison L, Kumar S, Bull M et al Clinical case series describes a contraindication for SerenoCem granules ${ }^{\mathrm{TM}}$ in mastoid obliteration: Our experience in sixty-four patients.Clinical Otolaryngology Allied Science 2017;42(5):1095-1100

13. Yung MW. The use of hydroxyapatite granules in mastoid obliteration. Clinical Otolaryngology Allied Science 1996;21(6):480-4

14. Munjal M, Passey J, Agarwal A et al. Hydroxyapatite granules for mastoid cavity obliteration: A study of 25 cases. TheInternet Journal of Otorhinolaryngology 2004;3:2

15. Yung MW, Karia KR. Mastoid obliteration with hydroyapatite - the value of high resolution CT scanning in detecting recurrent cholesteatoma. Clinical Otolaryngology Allied Science 1997;22(6):553-7

16. VercruysseJP, De Foer B, Somers T et al. Long-term follow-up after bony mastoid and epitympanic obliteration:radiological finding. Journal of Laryngology and Otology 2010;124(1):37-43

17. Kurien G, Greeff K, Gomaa N et al Mastoidectomy and mastoid obliteration with autologous bone graft:a quality of life study. Journal of Otolaryngology Head and Neck Surgery 2013;42(1):49

18. Hurley, R. and Iyer, A. (2020), Cavity obliteration in revision mastoidectomy leads to dry ear and improved quality of life: Our experience in 29 patients. Clin Otolaryngol, 45: 604-607. doi:10.1111/coa. 13532 

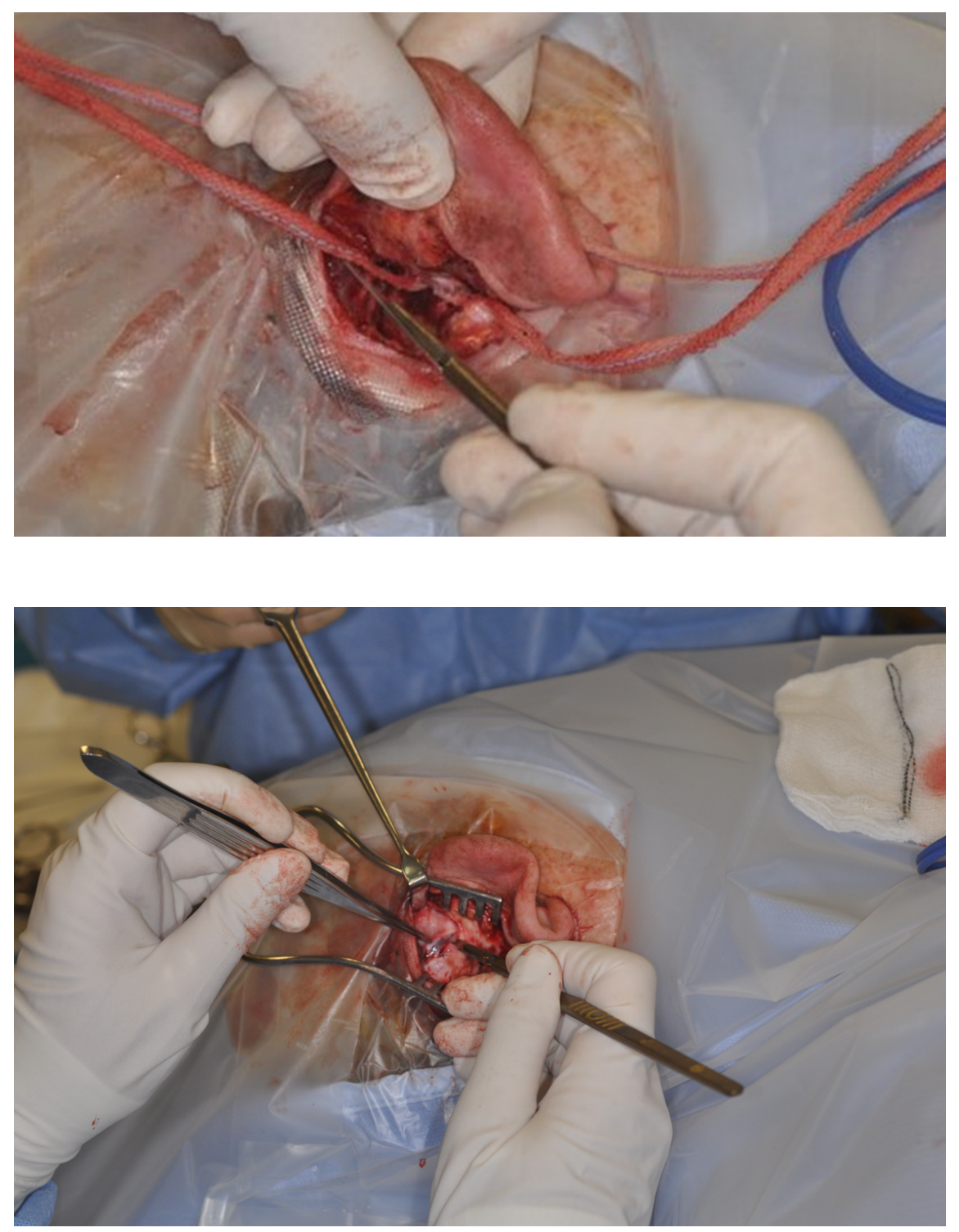

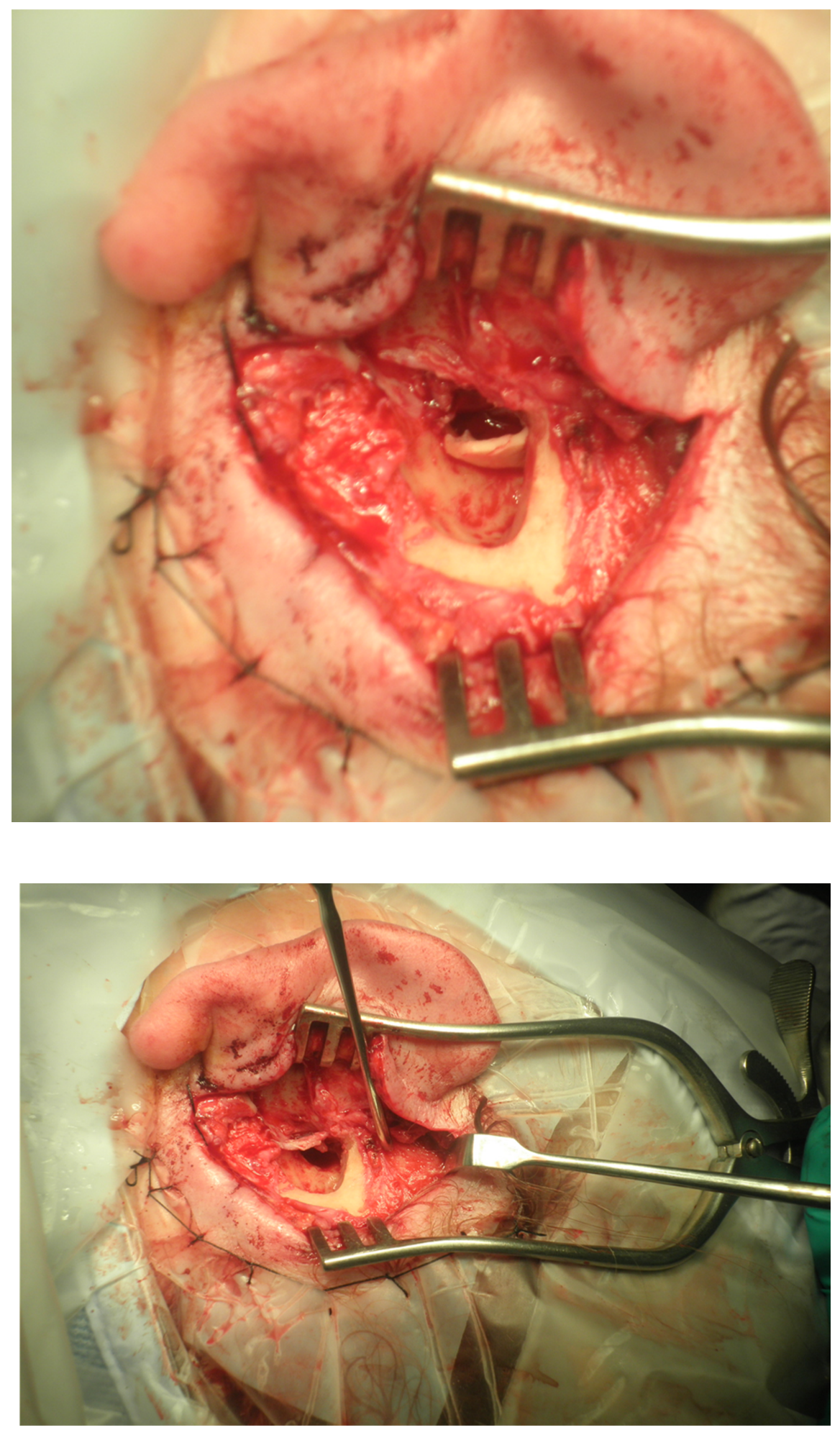

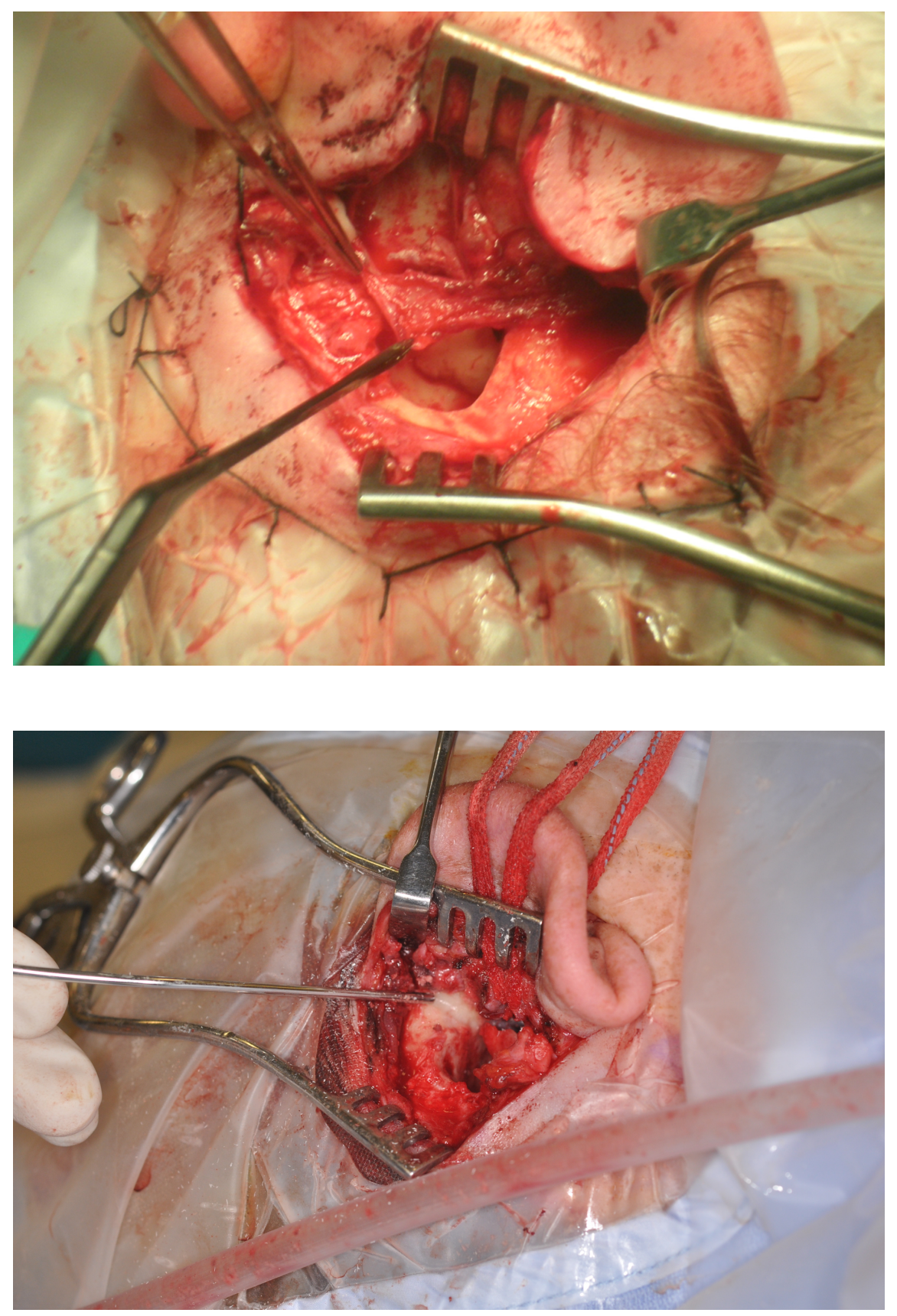

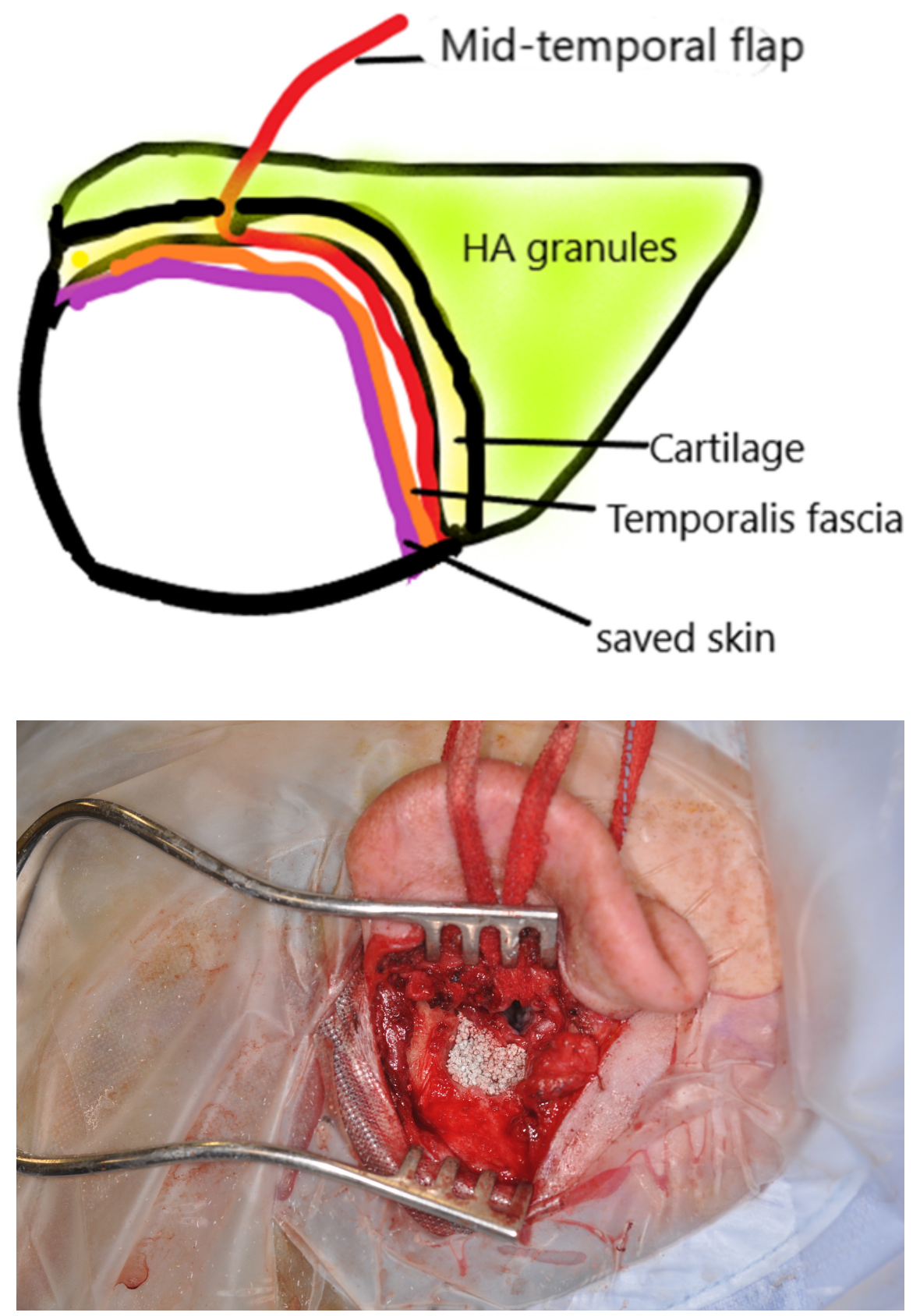

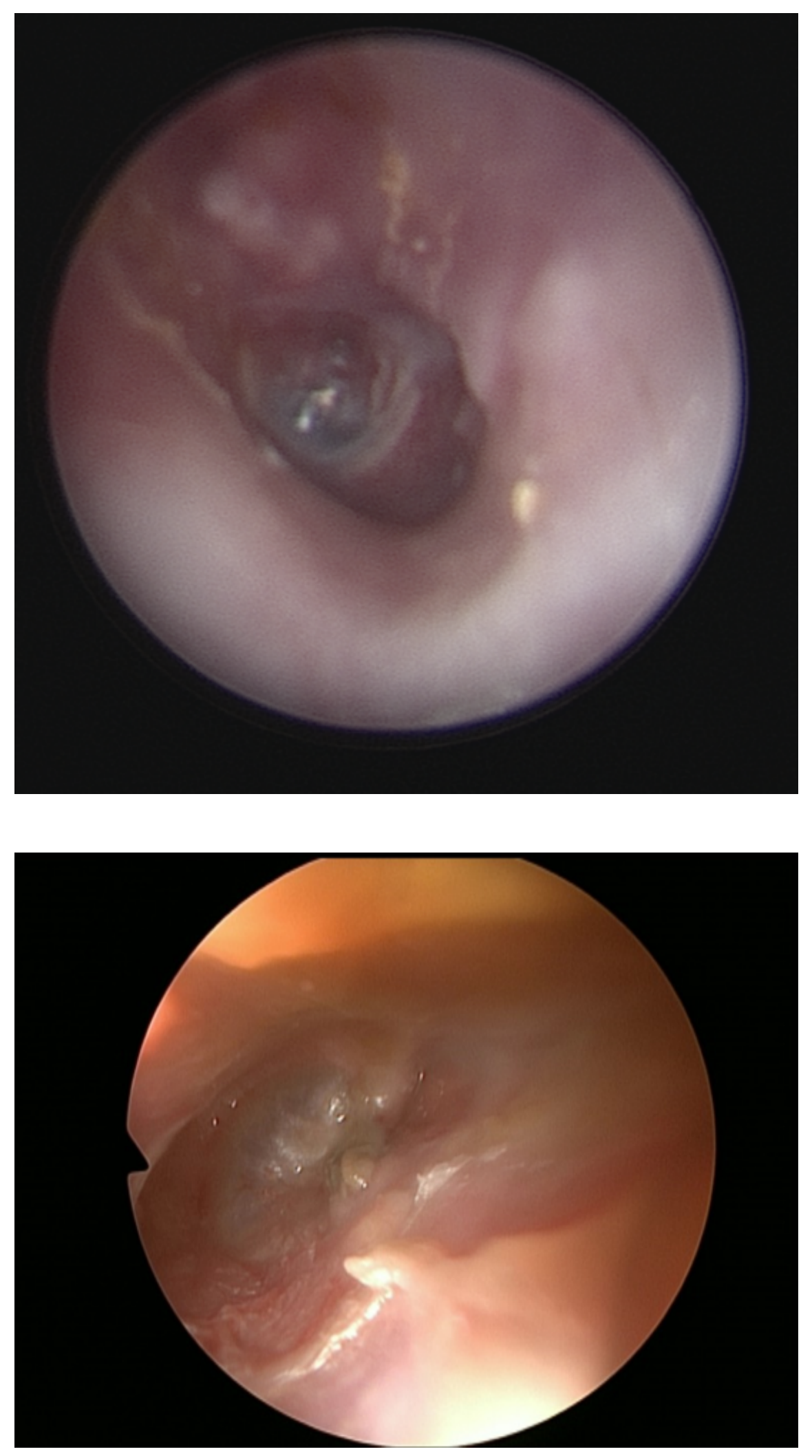


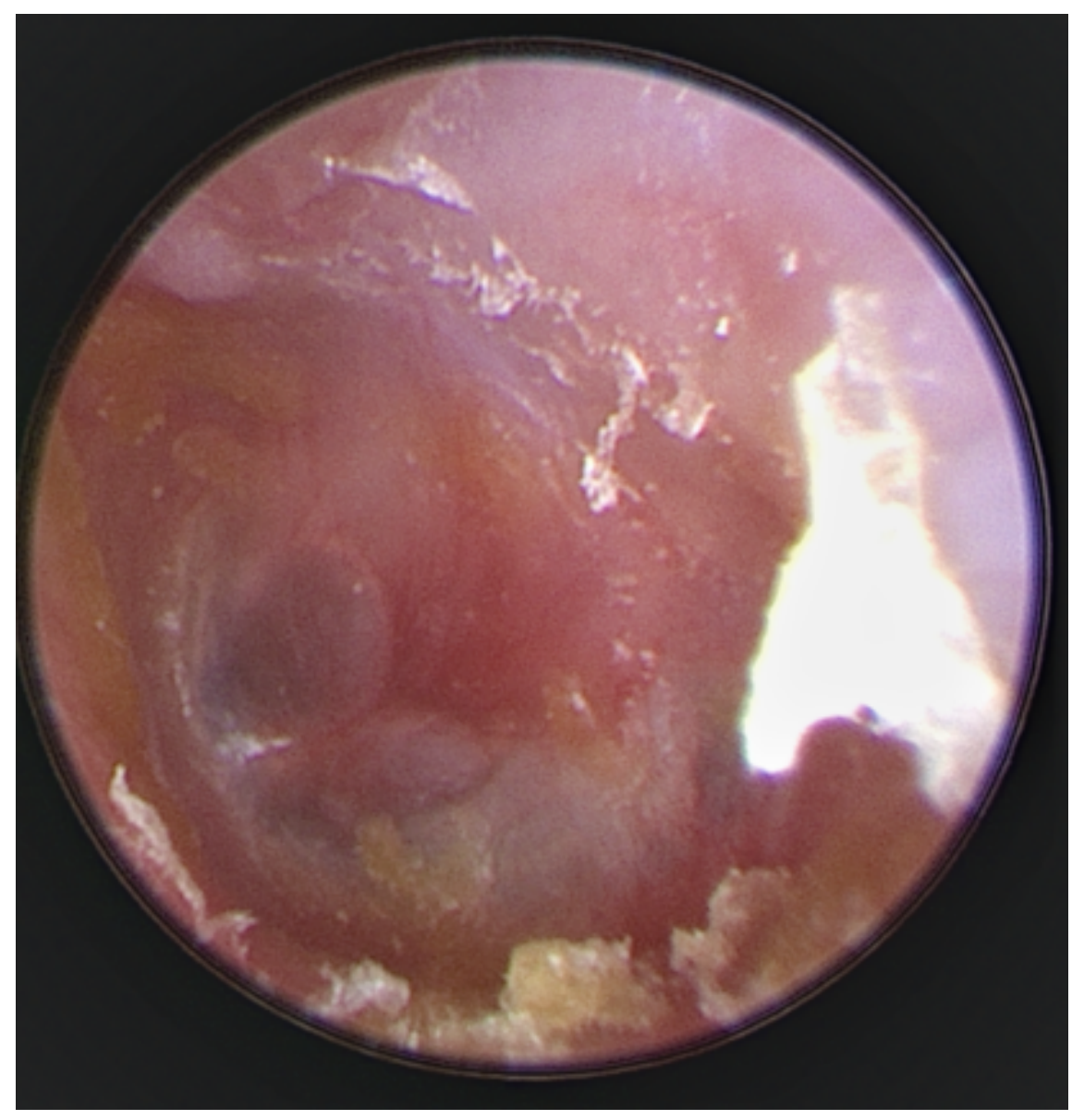

\section{Hosted file}

clin otol mo table 1.docx available at https://authorea.com/users/339886/articles/467638mastoid-obliteration-using-ha-granules-and-pericranial-flap-technique-and-results

\section{Hosted file}

clin otol mo table 2.docx available at https://authorea.com/users/339886/articles/467638mastoid-obliteration-using-ha-granules-and-pericranial-flap-technique-and-results 


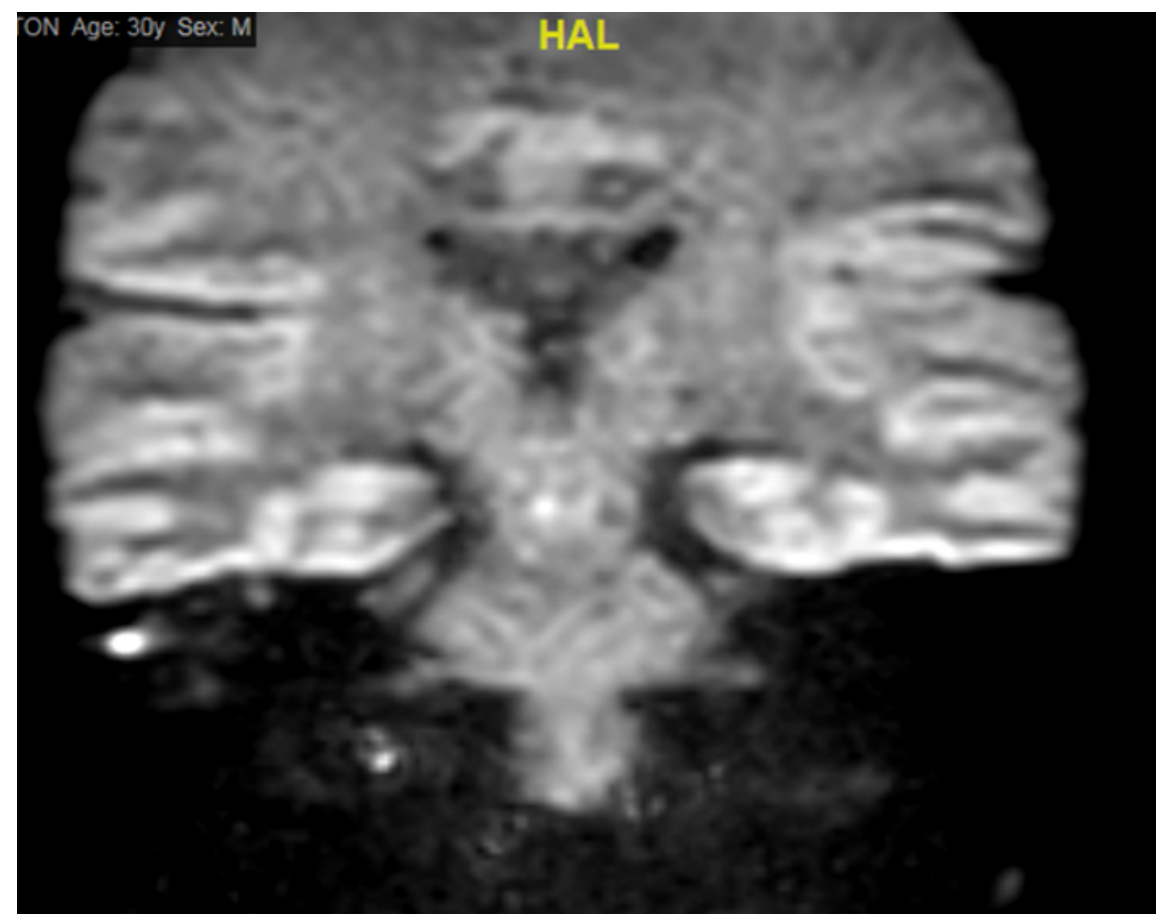

\section{Hosted file}

clin otol mo graph 1.docx available at https://authorea.com/users/339886/articles/467638mastoid-obliteration-using-ha-granules-and-pericranial-flap-technique-and-results

\section{Hosted file}

clin otol mo graph 2.docx available at https://authorea.com/users/339886/articles/467638mastoid-obliteration-using-ha-granules-and-pericranial-flap-technique-and-results

\section{Hosted file}

clin otol mo graph 3.docx available at https://authorea.com/users/339886/articles/467638mastoid-obliteration-using-ha-granules-and-pericranial-flap-technique-and-results

\section{Hosted file}

clin otol mo table 3.docx available at https://authorea.com/users/339886/articles/467638mastoid-obliteration-using-ha-granules-and-pericranial-flap-technique-and-results 